PROCEEDINGS OF THE

AMERICAN MATHEMATICAL SOCIETY

Volume 133, Number 6, Pages 1749-1756

S 0002-9939(04)07686-5

Article electronically published on December 31, 2004

\title{
SUBNORMAL SEMIGROUPS OF COMPOSITION OPERATORS
}

\author{
JOSÉ GIMÉNEZ
}

(Communicated by Joseph A. Ball)

\begin{abstract}
In this article we describe a model for subnormal semigroups of composition operators (with linear fractional symbol) acting on the Hardy space $H^{2}$. We also discuss cyclicity of such semigroups in the context of more general results studied by J. H. Shapiro and P. S. Bourdon.
\end{abstract}

\section{INTRODUCTION}

Let $A(\Delta)$ denote the space of all analytic functions in the unit disk $\Delta$ with the topology of uniform convergence on compact subsets of $\Delta$, and let $\mathcal{H}$ be a linear subspace of $A(\Delta)$. If $\psi$ is an analytic self-map of $\Delta$ such that $f \circ \psi$ belongs to $\mathcal{H}$ for all $f \in \mathcal{H}$, then $\psi$ induces a linear operator $C_{\psi}: \mathcal{H} \rightarrow \mathcal{H}$ defined as $C_{\psi}(f):=f \circ \psi \cdot C_{\psi}$ is called the composition operator with symbol $\psi$.

As usual, we will denote by $H^{2}$ the Hilbert space

$$
H^{2}(\Delta):=\left\{f \equiv \sum_{i=0}^{\infty} a_{i} z^{i} \in A(\Delta): \sum_{i=0}^{\infty}\left|a_{i}\right|^{2}<\infty\right\}
$$

where the vector operations are the pointwise ones, and the inner product is given by

$$
\left\langle\sum_{i=0}^{\infty} a_{i} z^{i}, \sum_{i=0}^{\infty} b_{i} z^{i}\right\rangle=\sum_{i=0}^{\infty} a_{i} \overline{b_{i}} .
$$

$H_{0}^{2}$ will denote the orthogonal complement of the constant functions in $H^{2}$; that is,

$$
H_{0}^{2}:=\left\{f \equiv \sum_{i=0}^{\infty} a_{i} z^{i} \in H^{2}: a_{0}=0\right\}=\left\{f \in H^{2}: f(0)=0\right\} .
$$

Definition 1.1. Let $\mathcal{H}$ be a Hilbert space. An operator $S \in \mathcal{L}(\mathcal{H})$ is said to be subnormal if there is a Hilbert space $\mathcal{K}$ containing $\mathcal{H}$ and a normal operator $N \in \mathcal{L}(\mathcal{K})$ such that $\left.N\right|_{\mathcal{H}}=S$. An operator $T \in \mathcal{L}(\mathcal{H})$ is said to be hyponormal if $T^{*} T \geq T T^{*}$.

In [10] the author pointed out that as a consequence of a result of C. C. Cowen (cf. [4]) if $\psi: \Delta \rightarrow \Delta$ is a linear fractional map, and the composition operator

Received by the editors November 8, 2002 and, in revised form, February 17, 2004.

2000 Mathematics Subject Classification. Primary 47B33; Secondary 47A13, 47B20.

This paper is based on some parts of the author's doctoral dissertation at the University of Iowa, written under the supervision of Professor Raúl Curto. The author has been partially supported by C.D.C.H.T. of the Universidad de Los Andes, project C - 1081-01- 05 - B. 
$C_{\psi}: H^{2} \rightarrow H^{2}$ is hyponormal, then $C_{\psi}$ is unitarily equivalent to a composition operator with symbol $\phi$ of the form

$$
\phi_{a}(z):=\frac{z}{a z+(a+1)}, \quad z \in \Delta, \quad a>0 .
$$

We also showed in [10] that if $\phi$ is a symbol of the form (1.1), then $\phi$ induces a strongly continuous one-parameter semigroup of subnormal composition operators. More precisely, we may define non-negative powers of $\phi_{a}$ by the formula

$$
\phi_{a}^{\lambda}:=\phi_{(1+a)^{\lambda}-1},
$$

and then put

$$
C_{\phi_{a}}^{\lambda}:=C_{\phi_{(1+a)^{\lambda}-1}} .
$$

Recall that if $\mathcal{H}$ is a Hilbert space, a function $A_{t}(t \in[0, \infty))$ from $[0, \infty)$ to the algebra of bounded operators on $\mathcal{H}$ is called a (one-parameter) semigroup of operators if

(i) $A_{t} A_{s}=A_{t+s}, \quad \forall t, s \in[0, \infty)$, and

(ii) $A_{0}=I$ (the identity operator on $\mathcal{H}$ ).

We will denote such a semigroup by $\left(\left\{A_{t}\right\}_{t>0}, \mathcal{H}\right)$. If it is clear from the context that $\left\{A_{t}\right\}_{t \geq 0} \subset \mathcal{L}(\mathcal{H})$, then we will refer to the semigroup simply as $\left\{A_{t}\right\}_{t \geq 0}$, or by a label such as $\mathcal{A}=\left\{A_{t}\right\}_{t \geq 0}$. The semigroup $\left\{A_{t}\right\}_{t \geq 0}$ is said to be strongly continuous if $\lim _{t \rightarrow s} A_{t}=A_{s}$ (in the strong operator topology) $\forall s \in[0, \infty$ ).

In fact, Theorem 2.5 in [10] can be restated as follows.

Theorem 1.2. Let $\boldsymbol{\Phi}=\left\{\phi_{s}\right\}_{s \geq 0}$ be a semigroup of linear fractional self-maps of $\Delta$, such that no $\phi_{s}$ has $\infty$ as a fixed point. The following statements are equivalent:

(i) $C_{\phi_{s}}$ is hyponormal for some $s \geq 0$.

(ii) $C_{\boldsymbol{\Phi}}$ is a strongly continuous semigroup of subnormal composition operators. Moreover, if (i) or (ii) hold, then there exists a sequence $\left\{\lambda_{i}\right\}_{i=1}^{n}$ of positive real numbers such that

$$
C_{\boldsymbol{\Phi}} \cong\left\{C_{\psi}^{\lambda_{i}}\right\} \quad \text { where } \psi(z):=\frac{1}{z+2} .
$$

The purpose of these notes is to exhibit a model, as a semigroup of multiplication operators, of $\left\{C_{\phi_{a}}^{\lambda}\right\}$, as well as to describe certain cyclicity properties of it.

\section{A MODEL FOR SUBNORMAL SEMIGROUPS OF OPERATORS}

The following is a well-known result due to T. Itô [12].

Theorem 2.1. Every strongly continuous semigroup $\left(\left\{A_{t}\right\}_{t \geq 0}, \mathcal{H}\right)$ of subnormal operators can be extended to a strongly continuous semigroup $\left\{N_{t}\right\}_{t \geq 0}$ consisting of normal operators acting on a Hilbert space $\mathcal{K}$ that contains $\mathcal{H}$.

Definition 2.2. A normal extension $\left(\left\{N_{t}\right\}_{t \geq 0}, \mathcal{K}\right)$ of $\left(\left\{A_{t}\right\}_{t \geq 0}, \mathcal{H}\right)$ is said to be minimal if $\mathcal{K}$ has no proper subspace that contains $\mathcal{H}$ and reduces each $N_{t}$. Equivalently, $\left(\left\{N_{t}\right\}_{t \geq 0}, \mathcal{K}\right)$ is the m.n.e. of $\left(\left\{A_{t}\right\}_{t \geq 0}, \mathcal{H}\right)$ if

$$
\mathcal{K}=\bigvee\left\{N_{t}^{*} \xi: t \in[0, \infty), \xi \in \mathcal{H}\right\}
$$

Theorem 2.3 (T. Itô). Any two minimal normal extensions (m.n.e.) of $\left(\left\{A_{t}\right\}_{t \geq 0}, \mathcal{H}\right)$ are unitarily equivalent. If $\left(\left\{N_{t}\right\}_{t \geq 0}, \mathcal{K}\right)$ is a minimal normal extension of $\left(\left\{A_{t}\right\}_{t \geq 0}, \mathcal{H}\right)$, then $\left\|N_{t}\right\|_{\mathcal{K}}=\left\|A_{t}\right\|_{\mathcal{H}}, \forall t \in[0, \infty)$. 
Remark 2.4. Let $\left\{N_{t}\right\}_{t \geq 0}$ be a strongly continuous semigroup of normal operators. Since the Fuglede-Putnam Theorem implies that $N_{t}$ commutes with $N_{s}^{*}$ for all $s, t \in[0, \infty)$, the selfadjoint operators $U_{t}:=N_{t}^{*} N_{t}$ also form a one-parameter semigroup.

Proposition 2.5. Let $\left\{N_{t}\right\}_{t \geq 0}$ be a strongly continuous semigroup of normal operators. Then the operators $N_{t}, t>0$, have one and the same null subspace.

Proof. It suffices to show that if $N_{s} f=0$, then $N_{u} f=0$ for $u>s$, and $N_{\frac{s}{2}} f=0$.

The first of these assertions follows from the identity $N_{u-s} N_{s}=N_{u}$. The second is a consequence of the relations

$$
\left\|N_{s} f\right\|^{2}=\left\langle N_{s}^{*} N_{s} f, f\right\rangle=\left\langle U_{s} f, f\right\rangle
$$

and

$$
\left\langle U_{s} f, f\right\rangle=\left\langle U_{\frac{s}{2}}^{2} f, f\right\rangle=\left\langle U_{\frac{s}{2}} f, U_{\frac{s}{2}} f\right\rangle=\left\|U_{\frac{s}{2}} f\right\|^{2} .
$$

Definition 2.6. A semigroup of operators $\mathcal{T}=\left\{T_{s}\right\}_{s>0}$ is said to be non-degenerate if the common null space $N(\mathcal{T})$ of the operators $T_{s}$ is $\{0\}$.

Proposition 2.7. If $\left\{N_{t}\right\}_{t \geq 0}$ is the minimal normal extension of a non-degenerate subnormal semigroup $\left\{S_{t}\right\}_{t \geq 0}$, then $\left\{N_{t}\right\}_{t \geq 0}$ itself is non-degenerate.

Proof. This follows from the definition of m.n.e., Theorem 2.3 and the fact that the kernel of a normal operator reduces it.

The following result also involves semigroups of normal operators (cf. [17]). A proof can be found in [14.

Theorem 2.8. Let $\left\{N_{t}\right\}_{t>0}$ be a non-degenerate, normalized (i.e., $\left\|N_{t}\right\|=1, \forall t>$ $0)$, strongly continuous semigroup of normal operators. Then $\left\{N_{t}\right\}_{t>0}$ has a unique spectral representation of the form:

$$
N_{t}=\int_{\Pi^{+}} e^{-t z} d E(z)
$$

where $\Pi^{+}:=\{z \mid \operatorname{Re}(z) \geq 0\}$ and $E(z)$ is the spectral measure on $\Pi^{+}$of the infinitesimal generator of the semigroup $\left\{N_{t}\right\}_{t>0}$.

Definition 2.9. A strongly continuous semigroup $\mathcal{T}=\{T(s)\}_{s \geq 0} \subset \mathcal{L}(\mathcal{H})$ is said to be cyclic if there is a vector $x_{0} \in \mathcal{H}$ such that $\bigvee\left\{T(s) x_{0}: s>0\right\}=\mathcal{H}$. The vector $x_{0}$ is said to be cyclic for $\mathcal{T}$. If there is family of vectors $\left\{x_{s}\right\}_{s>0} \subseteq \mathcal{H}$ such that $T(s) x_{t}=x_{s+t}, \forall s, t>0$, and $\bigvee\left\{x_{s}: s>0\right\}=\mathcal{H}$, then $\mathcal{T}$ is said to be quasicyclic, and the family $\left\{x_{s}\right\}_{s>0}$ is called a quasicyclic family for $\mathcal{T}$.

Using Theorem 2.8 we can slightly modify the proof of a result by R. Frankfurt ([9]) to establish our next result. We will need first the following definition.

Definition 2.10. Let $\Pi^{+}:=\{z \mid \operatorname{Re}(z) \geq 0\}$. Define a map $\tau$ from $\Pi^{+}$to the half-line $\{x \geq 0\}$ by $\tau(z):=\operatorname{Re}(z)$. If $\mu$ is a given measure on $\Pi^{+}$define a measure $\nu$ on $\{x \geq 0\}$ by $d \nu(x)=d \mu\left(\tau^{-1}(x)\right)$. The measure $\mu$ is said to have minimal exponential type if the Laplace-Stieltjes integral $\int_{0}^{\infty} e^{-s x} d \nu(x)$ converges for all $s>0$. In this case we have that $e^{-s z} \in L^{2}(\mu) \forall s>0$. Denote by $H^{2}(\mu)$ the $L^{2}(d \mu)$-closed linear span of these functions. 
Theorem 2.11. Let $\{T(s)\}_{s \geq 0}$, be a quasicyclic subnormal semigroup of contractions on a Hilbert space $\mathcal{H}$, with the property $\|T(s)\|=e^{k s}$ for all $s>0$, where $k=\ln \|T(1)\|$. Then there is a measure $\mu$ defined on the right halfplane $\Pi^{+}$and having minimal exponential type such that the semigroup $\{T(s)\}$ is unitarily equivalent to the semigroup of multiplication by $e^{s(k-z)}$ on $H^{2}(d \mu)$.

Proof. Let $\{N(s)\}_{s>0}$ be the minimal normal semigroup extension of the (normalized) semigroup $\left\{e^{-s k} T(s)\right\}_{s>0}$, acting on some Hilbert space $\mathcal{K} \supset \mathcal{H}$. Let $\left\{x_{s}\right\}_{s>0}$ be a quasicyclic family for $T(s)_{s>0}$. Then the minimality of $\{N(s)\}_{s>0}$ implies that it is non-degenerate, normalized (Theorem 2.3), and

$$
\mathcal{K}=\bigvee\left\{N(t)^{*} x_{s}: s, t>0\right\} .
$$

By Theorem 2.8, $N(s)$ has a spectral representation of the form

$$
N(s)=\int_{\Pi^{+}} e^{-s z} d E(z)
$$

where $E(z)$ is a spectral measure on $\Pi^{+}$. For each $s, t>0$, define a complex measure $\mu_{s, t}$ on $\Pi^{+}$by

$$
d \mu_{s, t}(z)=\left\langle d E(z) x_{s}, x_{t}\right\rangle .
$$

If $s=t$, we will write $\mu_{t, t}=\mu_{t}$. Then we have, for any $s, t, u, v>0$,

$$
d \mu_{s+u, t+v}=e^{(u z+v \bar{z})} d \mu_{s, t}(z) .
$$

Indeed, let $f$ be any continuous function with compact support in $\Pi^{+}$. Then

$$
\begin{aligned}
\int_{\Pi^{+}} f(z) d \mu_{s+u, t+v}(z) & =\int_{\Pi^{+}} f(z)\left\langle d E(z) x_{s+u}, x_{t+v}\right\rangle \\
& =\int_{\Pi^{+}} f(z)\left\langle d E(z) N(v)^{*} N(u) x_{s}, x_{t}\right\rangle \\
& =\left\langle\int_{\Pi^{+}} f(z) d E(z) \int_{\Pi^{+}} e^{-(u w+v \bar{w})} d E(w) x_{s}, x_{t}\right\rangle \\
& =\left\langle\int_{\Pi^{+}} f(z) e^{-(u z+v \bar{z})} d E(z) x_{s}, x_{t}\right\rangle \\
& =\int_{\Pi^{+}} f(z) e^{-(u z+v \bar{z})} d \mu_{s, t},
\end{aligned}
$$

which implies (2.2). In particular, for any $s, t>0$,

$$
d \mu_{s+t}(z)=e^{-2 s x} d \mu_{t}(z)(x=\operatorname{Re}(z)) .
$$

Hence

$$
d \mu(z):=e^{2 s x} d \mu_{s}(z) \quad(s>0)
$$

is a well-defined measure on $\Pi^{+}$. Moreover, since each $d \mu_{s}$ is finite, it follows that $d \mu$ has minimal exponential type. Let $\mathcal{H}_{0}=\operatorname{sp}\left\{N(t)^{*} x_{s}\right\}_{s, t>0}$, and define a transformation $U$ from $\mathcal{H}_{0}$ to $L^{2}(d \mu)$ by

$$
U\left(N(t)^{*} x_{s}\right)=e^{-(s z+t \bar{z})}
$$


for any $s, t>0$, and extend it by linearity to $\mathcal{H}_{0}$. Thus, if $s, t, u, v>0$,

$$
\begin{aligned}
\left\langle N(t)^{*} x_{s}, N(v)^{*} x_{u}\right\rangle_{\mathcal{K}} & =\left\langle N(t)^{*} N(v) x_{s}, x_{u}\right\rangle_{\mathcal{K}} \\
& =\int_{\Pi^{+}} e^{-(t \bar{z}+v z)}\left\langle d E(z) x_{s}, x_{u}\right\rangle \\
& =\int_{\Pi^{+}} e^{-(t \bar{z}+v z)} e^{-(s z+u \bar{z})} d \mu(z) \\
& =\int_{\Pi^{+}} e^{-(t \bar{z}+v z)} \overline{e^{-(u z+v \bar{z})}} d \mu(z) \\
& =\left\langle e^{-(s z+t \bar{z})}, e^{-(u z+v \bar{z})}\right\rangle_{L^{2}(d \mu)},
\end{aligned}
$$

which proves that $U$ is isometric. Hence $U$ extends to an isometric map from $\mathcal{K}$ to $L^{2}(d \mu)$. In particular, since $U\left(x_{s}\right)=e^{-s z}, \forall s>0, U$ carries $\mathcal{H}$ isometrically onto $H^{2}(d \mu)$. It is readily seen that $U$ intertwines the semigroup $\left\{e^{-k} T(s)\right\}_{s>0}$, and the semigroup of multiplication by $e^{-s z}, s>0$. The result now follows.

\section{CyCLICITY}

P. S. Bourdon and J. H. Shapiro have exhaustively studied and described the cyclic behavior of linear fractional composition operators in their book Cyclic Phenomena for Composition Operators [3]. Recall that an operator $T$ in a Hilbert space $\mathcal{H}$ is said to be cyclic if there is a vector $x \in \mathcal{H}$ whose orbit

$$
\operatorname{Orb}(T, x):=\left\{T^{n} x: n=0,1, \ldots\right\}
$$

has dense linear span. $T$ is called hypercyclic if there is $y \in \mathcal{H}$ such that $\overline{\operatorname{Orb}(T, y)}=\mathcal{H}$.

As usual $x$ (resp. $y$ ) is called a cyclic (resp. hypercyclic) vector for $T$.

In [3] the reader can find a proof of the following fact: an operator has either no hypercyclic vector or a dense $G_{\delta}$ set of them. As a consequence, Baire's theorem implies: Every countable collection of hypercyclic operators has a common hypercyclic vector. On the other hand, [2, Theorem 2.8] implies that for any $\lambda>0,\left.C_{\phi_{a}}^{\lambda}\right|_{H_{0}^{2}}$ is not cyclic (as a single operator), and that, in fact, the closed linear span of any orbit of $\left.C_{\phi_{a}}\right|_{H_{0}^{2}}$ has infinite codimension.

Now, cyclicity (resp. hypercyclicity) deals with the fact that the set obtained by applying the integer powers of $T$ to a cyclic (resp. hypercyclic) vector, has dense linear span (resp. is dense); however, in our case we have more powers of $\left.C_{\phi_{a}}\right|_{H_{0}^{2}}$ in which to rely, and this might turn out to produce a better cyclic behavior in a certain sense.

Definition 3.1. A Hilbert space $\mathcal{H}$ of analytic functions defined on an open region $\Omega \subset \mathbb{C}$ is called a functional Hilbert space if for each $z \in \Omega$ the linear functional $f \mapsto f(z)$ is continuous. In this case, the Riesz representation theorem implies that for each $z \in \Omega$ there is a function $\mathbf{K}_{z} \in \mathcal{H}$, called a reproducing kernel, such that

$$
f(z)=\left\langle f, \mathbf{K}_{z}\right\rangle .
$$

One important step in establishing the hyponormality of $C_{\phi_{a}}: H^{2} \rightarrow H^{2}$ is to consider the restriction $\left.C_{\phi_{a}}\right|_{H_{0}^{2}}$. It is readily seen, on the other hand, that both $H^{2}$ and $H_{0}^{2}$ are functional Hilbert spaces, and since the set $\left\{z, z^{2}, \ldots,\right\}$ is an 
orthonormal basis for $H_{0}^{2}$, it follows (cf. [1]) that the reproducing kernels of $H_{0}^{2}$ are the functions

$$
K(z, w):=K_{w}(z)=\sum_{n=1}^{\infty} z^{n} \bar{w}^{n}=\frac{\bar{w} z}{1-\bar{w} z}, \quad z, w \in \Delta .
$$

Notice that the kernels $K_{w}$ are linear fractional self-maps of $\Delta$. Moreover, the symbols $\left\{\phi_{a}^{\lambda}\right\}$ are, in fact, scalar multiples of these kernels. Indeed,

$$
\phi_{a}(z)=\frac{z}{a z+1+a}=-\frac{1}{a} \frac{\left(-\frac{a}{1+a}\right) z}{1+\left(\frac{a}{1+a}\right) z}=-\frac{1}{a} K_{\left(-\frac{a}{1+a}\right)} .
$$

On the other hand, it is well known (cf. [5) that the adjoint of any composition operator $C_{\phi}: \mathcal{H} \rightarrow \mathcal{H}$, where $\mathcal{H}$ is a functional Hilbert space contained in $A(\Delta)$, satisfies: $C_{\phi}^{*} \mathbf{K}_{w}=\mathbf{K}_{\phi(w)}$, for all $w \in \Delta$.

Notice that if $u_{s}:=\phi_{a}^{s}, s \geq 0$, then $C_{\phi_{a}}^{t} u_{s}=u_{t+s}$. The following proposition shows that the set $\left\{u_{s}\right\}_{s>0}$ actually spans $H_{0}^{2}$.

Proposition 3.2. Let $T(s):=\left.C_{\phi_{a}}^{s}\right|_{H_{0}^{2}}, s \geq 0$. For all $t \geq 0$, the function $u_{t}:=\phi_{a}^{t}$ is a cyclic vector for the semigroup $\{T(s)\}$.

Proof. Fix $t \in[0, \infty)$. Suppose that $g \in H_{0}^{2}$ is orthogonal to $\bigvee\left\{T(s) u_{t}: s \geq 0\right\}$; then

$$
\begin{aligned}
0 & =\left\langle g, T(s) u_{t}\right\rangle \\
& =\left\langle g, u_{s+t}\right\rangle \\
& =\frac{-1}{(1+a)^{(s+t)}-1}\left\langle g, K_{\left\{-\frac{(1+a)(s+t)-1}{(+a)^{(s+t)}}\right\}}\right\rangle \\
& =\frac{-1}{(1+a)^{(s+t)}-1} g\left(\frac{1}{(1+a)^{(s+t)}}-1\right) .
\end{aligned}
$$

Therefore, the analytic function $g$ vanishes along the curve $\left\{\frac{1}{(1+a)^{(s+t)}}-1, s \geq 0\right\} \subset$ $\Delta$, which means $g \equiv 0$.

Remark 3.3. In general, for a strongly continuous semigroup $\{T(s)\}_{s \geq 0}$, there are constants $w \in \mathbb{R}$ and $M \geq 1$ such that $\|T(s)\| \leq M e^{w s}$ (cf. [13], Proposition I.5.5). The semigroup $\left\{\left.C_{\phi_{a}}^{s}\right|_{H_{0}^{2}}\right\}_{s \geq 0}$, however, satisfies

$$
\left\|\left.C_{\phi_{a}}^{s}\right|_{H_{0}^{2}}\right\|=\left((1+a)^{-\frac{1}{2}}\right)^{s}=e^{s \ln \left\|\left.C_{\phi_{a}}\right|_{H_{0}^{2}}\right\|}
$$

cf. [10, Theorem 2.14].

As a consequence of the preceding discussion we have

Theorem 3.4. The semigroup $\{T(s)\}_{s \geq 0}$ is unitarily equivalent to the semigroup of multiplication by $e^{s(\ln \|T(1)\|-z)}$ on $H^{2}(\mu)$, where $\mu$ is a measure defined on $\Pi^{+}$, having minimal exponential type.

Now we establish a general result on quasicyclicity of the adjoint of a semigroup of composition operators.

Theorem 3.5. Let $\Phi:=\{\phi(s)\}_{s \geq 0}$ be a semigroup of analytic self-maps of $\Delta$, such that $\phi(s)$ has no fixed points in $\Delta$, for $s \neq 0$. Then the adjoint, $\left\{C_{\phi(s)}^{*}\right\}_{s>0} \subseteq$ $\mathcal{L}\left(H^{2}\right)$, of the semigroup of composition operators induced by $\Phi$, is quasicyclic. 
Moreover, for any non (eventually) constant convergent sequence $\left\{s_{k}\right\} \subseteq[0, \infty)$, there are infinitely many vectors $f \in H^{2}$ such that

$$
\bigvee\left\{C_{\phi\left(s_{k}\right)}^{*} f: k>0\right\}=H^{2}
$$

Proof. Pick any $w \in \Delta$, and let $x_{s}:=K_{\phi(s)(w)}\left(=C_{\phi(s)}^{*} K_{w}\right)$. Then

$$
\begin{aligned}
C_{\phi(t)}^{*} x_{s} & =C_{\phi(t)}^{*} C_{\phi(s)}^{*} K_{w_{0}} \\
& =C_{\phi(t+s)}^{*} K_{w} \\
& =K_{\phi(t+s)}(w) \\
& =x_{t+s} .
\end{aligned}
$$

The hypothesis on $\Phi$ guarantees that the relation

$$
s \longrightarrow \phi(s)(w)
$$

defines a simple smooth curve contained in $\Delta$; indeed, suppose $t>s$ and that $\phi(t)(w)=\phi(s)(w)$. Then $\phi(s) \circ \phi(t-s)(w)=\phi(s)(w)$, which implies (since $\phi(t)$ is univalent) that $\phi(t-s)(w)=w$ and therefore (since $\phi(t-s)$ has no fixed points in $\Delta)$ that $t=s$. Thus if $g \in H^{2}$, then

$$
\begin{aligned}
\left\langle g, x_{s}\right\rangle=0 \quad \forall s & \Leftrightarrow\left\langle g, C_{\phi(s)}^{*} K_{w}\right\rangle=0 \quad \forall s \\
& \Leftrightarrow g(\phi(s)(w))=0 \quad \forall s \\
& \Leftrightarrow g \equiv 0,
\end{aligned}
$$

by the uniqueness principle for analytic functions. This argument shows that $\left\{C_{\phi(s)}^{*}\right\}_{s>0}$ is quasicyclic. The last assertion can be proved similarly (by putting $\left.f:=K_{w}\right)$.

Remarks 3.6.

- Actually, Proposition 3.2 can be deduced from Theorem 3.5 due to the fact that $\left.C_{\phi_{a}}\right|_{H_{0}^{2}}=s M_{z} C_{\sigma_{a}}^{*} M_{\frac{1}{z}}$, where $\sigma_{a}(z)=s z+s-1$ with $s=\frac{1}{1+a}$, and $M_{z}$ and $M_{\frac{1}{z}}$ denote the operators of multiplication by $z$ on $H^{2}$ and by $\frac{1}{z}$ on $H_{0}^{2}$, respectively; see [4].

Thus, the semigroup $\left\{\left.C_{\phi_{a}}^{\lambda}\right|_{H_{0}^{2}}\right\}_{\lambda>0}$ is unitarily equivalent to a semigroup of the form $\left\{s^{t} C_{\sigma(t)}^{*}\right\}_{t>0}$, where $\{\sigma(t)\}$ is a semigroup of analytic self-maps of $\Delta$ such as the one described in Theorem 3.5

Note also that if $\left\{S_{t}\right\}$ is a quasicyclic subnormal semigroup, $k$ is a positive constant, and we define

$$
T_{t}:=k^{t} S_{t} \quad(t>0),
$$

then $\left\{T_{t}\right\}_{t>0}$ is also a quasicyclic subnormal semigroup.

- Applying Theorem [3.5 to the semigroup $\left\{\phi_{a}^{\lambda}\right\}$, equation (3.2) turns into

$$
\bigvee\left\{\left(C_{\phi_{a}}\right)^{* s_{k}} f: k>0\right\}=H^{2}
$$

which resembles cyclicity.

In [9] R. Frankfurt points out the fact that if the measure $\mu$, given in Theorem 2.11 is finite, then $U^{*}(1)$ is a cyclic vector for $\{T(s)\}_{s>0}$. He also gives an example that the converse, in general, is not true. In the present situation, however, we have

Corollary 3.7. The measure $\mu$, corresponding to $\left\{\left.e^{-s k} C_{\phi_{a}}^{s}\right|_{H_{0}^{2}}\right\}_{s \geq 0}$ by Theorem [2.11, is a probability measure. 
Proof. It is readily seen that if $w=0$ in the proof of Theorem [3.5, then for all $\lambda, \mu>0$,

Hence

$$
\left\langle x_{\lambda}, x_{\mu}\right\rangle_{H_{0}^{2}}=\frac{1}{1-\left(s^{\lambda}-1\right)\left(s^{\mu}-1\right)} .
$$

$$
\int_{\Pi^{+}} d \mu=\langle 1,1\rangle_{H^{2}(d \mu)}=\left\langle x_{0}, x_{0}\right\rangle_{H_{0}^{2}}=1
$$

I would like to thank Professor Raúl Curto for his valuable suggestions. I am also indebted to the referee of the previous versions of this paper who made several recommendations to improve the exposition.

\section{REFERENCES}

[1] N. Aronszajn, Theory of reproducing Kernels, Trans. Amer. Math. Soc. 68 (1950), 337-404. MR.0051437(14:479c)

[2] E. Berkson and H. Porta, Semigroups of analytic functions and composition operators, Michigan Math. J. 25 (1978), 101-115. MR0480965 (58:1112)

[3] P. S. Bourdon and J. H. Shapiro, Cyclic Phenomena for Composition Operators, Memoirs of the American Mathematical Society, 1997. MR.1396955 (97h:47023)

[4] C. C. Cowen, Linear fractional composition operators on $H^{2}$, Integral Equations and Operator Theory 11 (1988), 151-160. MR0928479 (89b:47044)

[5] C. C. Cowen, and B. D. MacCluer, Composition Operators on Spaces of Analytic Functions, CRC Press, New York, 1994. MR1397026 (97i:47056)

[6] C. C. Cowen, and T. L. Kriete, Subnormality and composition operators on $H^{2}$, J. Functional Analysis 81 (1988), 298-319. MF0971882 (90c:47055)

[7] R. Curto, P. Muhly and J. Xia, Hyponormal pairs of commuting operators, Operator Theory: Advances and Applications, Vol. 35, Birkhäuser Verlag, Basel (1988), 1-21. MR1017663 (90m:47037)

[8] F. Jafary, B. D. MacCluer, C. C. Cowen and D. Porter, Studies on Composition Operators, Memoirs of the American Mathematical Society, 1997. MR 1601044 (98h:47005)

[9] R. Frankfurt, Quasicyclic subnormal semigroups, Canadian Journal of Mathematics, vol XXIX, 6, (1977), 1230-1246. MR0463971 (57:3909)

[10] J. Giménez, Joint hyponormality of composition operators with linear fractional symbols, Integral Equations and Operator Theory 43 (2002), 385-396. MR.1909372 (2003e:47049)

[11] J. Giménez, Joint spectrum of subnormal n-tuples of composition operators, Proc. Amer. Math. Soc. 130 (2001), 2015-2023. MF,1896036 (2003b:47045)

[12] T. Itô, On the commutative family of subnormal operators, J. Fac Sci. Hokkaido University, 14 (1958), 1-15. MR0107177 (21:5902)

[13] K-J. Engel and R. Nagel, One-Parameter Semigroups for Linear Evolution Equations, Grad. Texts in Math., 194, Springer-Verlag, 2000. MR.1721989 (2000i:47075)

[14] A. I. Plesner, Spectral Theory of Linear Operators, Vol II, Frederick Ungar Publishing Co., New York, 1969. MR0244792 (39:6106)

[15] J. H. Shapiro, Composition Operators and Classical Function Theory, Springer-Verlag, New York, 1993. MR 1237406 (94k:47049)

[16] A. G. Siskakis, Semigroups of composition operators on spaces of analytic functions, a review, Studies on Composition Operators, Contemporary Math, AMS, 213, 1988, 229-252. MR.1601120 (98m:47049)

[17] B. Sz.-Nagy, Spektraldarstellung linearer transformationen des Hilbertschen raumes, Ergebnisse der Mathematik, Springer-Verlag, 1967. MR0213890|(35:4744)

Departamento de Matemáticas, Facultad de Ciencias, Universidad de los Andes, MÉrida, Venezuela

E-mail address: jgimenez@.ula.ve 